\title{
Acknowledgement of Reviewers for 2019
}

\author{
Petra van Steenbergen ${ }^{1}$
}

Published online: 29 January 2020

(c) Institute of Geophysics, Polish Academy of Sciences \& Polish Academy of Sciences 2020

We thank all those scientists and experts in the various fields represented in Acta Geophysica for devoting their time and effort to review the papers that we have sent them. The Editor in Chief, and the Publisher acknowledge the colleagues listed below for their most helpful reviews of papers which have been provided during the period January 2019 to December 2019.

\begin{tabular}{ll} 
Mubarik Ali & Mohammad Khaled Akhtar \\
Victor Aarre & John-Felix Akinbami \\
Ivan Abakumov & Halil Akinci \\
Abdulaziz M. Abdulaziz & Mohammed Al Yafei \\
Karimatu Lami Abdullahi & Hakan Alçik \\
Sumiyoshi Abe & Maria Joao Alcoforado \\
Jahangir Abedi-Koupai & Lorenzo Alfieri \\
Jochen Aberle & Syed Shahid Ali \\
O. Abiola & Tariq Alkhalifah \\
Raymond Abma & Mostafa AllamehZadeh \\
Ahmed E. Abouelregal & Mansour Almazroui \\
Yousf Abushalah & Ibrahim A. Almosallam \\
Sirisha Adamala & David Altadil \\
Jan Adamowski & Orhan Altan \\
Alice Adenis & Nezih Altay \\
Tjahyo Nugroho Adji & Luis Alva Valdivia \\
Muhammad Adnan & Hakimeh Amanipoor \\
Qazi Adnan Ahmad & A. Amendola \\
M. H. Afshar & John Amigun \\
Amir AghaKouchak & Babak Amirataee \\
Afshin Aghayan & Angelo Amorosi \\
Babak Aghel & Amsalu Y. Anagaw \\
Zacharias Agioutantis & Emmanouil Anagnostou \\
Mehdi Aharchaou & Marios Anagnostou \\
Mobin-ud-Din Ahmad & Anca Radulescu \\
Waqar Ahmad & Amalia Anderson \\
Ali Ahmadalipour & Karin Andreassen \\
Hassan Ahmadi & Gina Andrei \\
Alireza Ahmadyfard & Jesse Andries \\
Jamilu Bala Ahmed II & D. A. Angus \\
Valentin Aich & Marco Anzidei \\
Mehdi Akhoondzadeh & George Apostolopoulos \\
& \\
\hline &
\end{tabular}

Alireza Arabameri
Mustafa Aral
Frederic Arand
David Archer
Mehran Arian
Feza Arikan
Keisuke Ariyoshi
Endrias Asgedom
Siavash Ashoori
Aysegul Askan
Elvira I. Astafyeva
Ariana Luci Astorga
Barbara Atamaniuk
Mustafa Mujdat Atanak
Rahman Atta-ur
Pascal Audet
Esben Auken
Christine Authemayou
Dmitry Avdeev
Anna Avdeeva
Mekonen Ayana
Naser Ayat
Hossein Azizi
Alper Baba
Golekar Rushikesh Baburao
Jose Badal
Marcela Elvira Baena Rivera
Nooshin Bagha
Majid Bagheri
Dov Bahat
Christopher Baiyegunhi
Marianna Balasco

Petra van Steenbergen

petra.vansteenbergen@springer.com

1 Dordrecht, The Netherlands 
Huang Baochun

Simone Barani

Sandor Baranya

Reza Barati

Gabriel Barberes

Udo Barckhausen

Adrian S. Barfod

Brandon Barkey

Eric Barrier

Roland Barthel

Rahim Barzegar

Jelena Basarić

Stefano Basso

Paul Bates

George Bathrellos

Joelson Batista

Pedro V. G. Batista

Rajan Batta

Michael L. Batzle

D. Bau

Stanisław Baudzis

Carlos Bautista-Capetillo

Paolo Bazzurro

Hylke Beck

L. Becker

Simon Beckouche

Jonathan Bedford

J. Bedi

Michael Behm

Linus Bengtsson

Martin Beniston

Djillali Benouar

Jens Berdermann

Francoise Bergerat

Sten Bergstrom

James G. Berryman

Falko Bethmann

Vittal Bhatt

Shuvajit Bhattacharya

Muhammad Tousif Bhatti

Hussain Bux Bhutto

P. K. Bhuyan

Huaxing Bi

Pier Francesco Biagi

Robert Bialik

Vanessa Biando Ribeira

Ramón Bienes

Dieter Bilitza

Ahmad Safuan Bin A Rashid

Dino Bindi

Peter Bird

Arkoprovo Biswas

Andrea Bizzarri
Simone Bizzi

Viktória Blanka

Lilian Blaser

Leszek Blaszkiewicz

Jan Blecki

Fred Boadu

Nikita Bobrov

Heike Bock

Ewa Bogdanowicz

Jedrzej Bojanowski

Bodo Bookhagen

David Boore

Angana Borah

Marco Borga

José Fernando Borges

Ronaldo Borja

Jannis Born

Pasquale Borrelli

Maren Böse

Ada Vittoria Bosisio

Josef Boska

Gianluca Botter

Hamza Bouguerra

Claire Bouligand

Polyzois Bountzis

Housseyn Bouzeria

Heinrich Brasse

Rebecca Briant

Alistair R. Brown

Holly M. Brown

Dominik Brunner

Manuela Brunner

Pace Bruno

Axel Bruns

Sander Bruun

S. V. Bukharin

Dalia Buresova

Gerd Bürger

Roland Bürgmann

Vyacheslav Burov

Michael Bush

Martina Bussettini

P. Butlin

Jimmy Byakatonda

Joanna Bzówka

Tommaso Caloiero

A. Campisano

Iza Canales García

Ljiljana Cander

Jingjie Cao

Philippe Caprioli

Roberto Carluccio

Chris Caroni
David Carpenter

Armando Carrillo-Vargas

Fernando Carvajal-Ramírez

John P. Castagna

Silvia Castellaro

Andrea Castelletti

Raúl R. Castro

Marcilio Castro de Matos

Alessandro Cattapan

Carlo Cauzzi

Daniel Caviedes-Voullième

Alberto Cazzella

Semet Celik

A. M. Chandler

Vishal Chandole

Emmy Chang

Liang-Cheng Chang

Ping-Yu Chang

Da-Yi Chen

Jin Chen

Ke Chen

Ling Chen

Shengbo Chen

Shengchang Chen

Yangkang Chen

You-Cheng Chen

Yuanfang Chen

Ismail Chenini

Piyaphong Chenrai

Mohsen Cheraghi

Dmitri B. Chklovskii

Hsiao Chi Chuang

Chen Chieh-Hung

Francesco Chierici

Karem Chokmani

Richard Chopping

Konstantinos Chousianitis

Jinder Chow

Jasti S Chowdary

Vasileios Christelis

Craig William Christensen

Maria Christodoulou

Hone-Jay Chu

Artur Cichowicz

Consuelo Cid

Luigi Cimorelli

Begüm Civgin

Riccardo Civico

Pierluigi Claps

Elizabeth S Cochran

Richard E. LL. Collier

Andrea Cominola

Christian Conoscenti 
Rodolfo Console

R. Corthesy

Giacomo Corti

Sara Corvaro

Stephen Costabel

Alex Costall

Carmine Covelli

Corina Coviaga

Stephan Creelle

Gaetano Crispino

Alessandra Crosato

Russell Crosbie

Brendan W. Crowell

Marcin Cudny

Armando Cuéllar

Gloria Curilem

Joseph Curray

Marta Cyz

Rafał Czarny

Zbigniew Czechowski

Federico Da Col

Adisa Daberdini

Nicola D'Agostino

Berit Dahl

Torsten Dahm

Minglong Dai

Phil Dales

Irina Dallo

Ali Danandeh Mehr

Tomasz Danek

Diaconu Daniel

Andrea D'Aniello

Sandipan Das

Rachel Davidson

Ammar Dawood

Shanker Daya

Sikhhendra De

Inge de Graaf

Carmen De Jong

Mariano Moreno de las Heras

Paolo Marco De Martino

Riccardo De Ritiss

Angelo De Santis

Luca de Siena

Jerzy Dec

E. J. van Dedem

M. Dehbozorgi

Nicholas Deichmann

Manuel del Jesus

B. Delouis

Mehmet Demirel

Nihan Çetin Demirel

Tufan Demirel
Matteo Demurtas

Muhammet Deveci

Jacques Déverchère

Roberto Devoti

Subhasish Dey

Amit Dhorde

Dirk Diederen

Panayiotis Dimitriadis

Radu Georgiu Dimitriu

Savka Dineva

Pinbo Ding

Antonino D'Ippolito

Son Doan

Mihály Dobróka

Longjun Dong

Sheen Dong-Hoon

R. D. Dony

Mahmut Drahor

Krzysztof Drozdowicz

Changwen Du

Qingyun Duan

Matthieu Dufresne

Stanica Dumitru

Victor Dupuis

Ved Vyas Dwivedi

Tomasz Dysarz

Beata Dziak-Jankowska

Y. Ebert

Mohsen Ebrahimi

Daniele Ehrlich

Leo Eisner

Salamatu Abraham Ekpo

John Elliott

Sherif A. Abu El-Magd

H. M. El-Sayed

Ahmed El-Shafie

Mustafa Erdik

Semih Ergintav

Tomasz Ernst

Thebault Erwan

Khalid Essa

Mark Everett

Yener Eyüboğlu

Licia Faenza

Kin Fai Ho

Maria Rosaria Falanga

Giuseppe Falcone

Ke Fan

Ying Fan

Chunhui Fang

Xinding Fang

Mohammed Farfour

Shahab Fazal
Maurizio Fedi

Carozzi Fernanda

Vito Ferro

Gianluca Fiandaca

Stephen Fick

F. Fiedrich

Julie S. Field

Peter Fiener

Ilias Fikos

Renato Filjar

Ruhul Firdaus

Ms Fiza Sarwar

Jan Fleckenstein

Michael Floyd

Stéphane Follain

Elizabeth Follett

Sergey Fomel

lluis Font

Ioanna-Kleoniki Fontara

J. Fontiela

Clive Foss

Dimitra Founda

Paolo Franchin

Anna Franczyk

Gabriele Freni

Vladimir Frid

Horst Frischkorn

Vinay Kumar Gadi

Stefano Galelli

Ivan Galkin

Chiara Galletti

Danilo Galluzzo

Attila Galsa

Sreedhar Ganapuram

Jinghuai Gao

Jianjun Gao

Huiji Gao

Yanhong Gao

Pablo Garcia

Amanda García Marín

Daniel Garcia-Castellanos

Akhil Garg

Evangelia Garini

Dariusz Gąsiorowski

Paolo Gasperini

Mohammed Gedefaw

Nina Gegenhuber

Violette Geissen

Alexandra Gemitzi

Stefania Gentili

Roger Ghanem

Aminuddin Ab Ghani

Mohammad Ali Ghannad 
Amin Gharehbaghi

A. Ghasemi

Mohammad Ghayamghamian

Lubna Ghazal

Abdul Halim B. Ghazali

Ali Gholami

Mohammad Ghorbani

Gopal Ghosh

Sayantan Ghosh

Abolfazl Giglou

Ljubomir Gigović

Stuart Gilder

Eric Gilleland

Agnieszka Gil-Swiderska

Dorota Giriat

Ruediger Glaser

Milan Gocic

Bettina Goertz-Allmann

Avto Gogichaishvili

Tomisław Gołębiowski

Ali Golkarian

Gennady Goloshubin

Abolfazl Golshan

Francisco Gomez

Javier Gomez

David Gomez-Ortiz

Xiangbo Gong

Marjan Goodarzi

Mustafa Goodarzi

Kazım Görgülü

Andrzej Górszczyk

Ajanta Goswami

Yozo Goto

Teresa Grabowska

Dariusz Graczyk

Roy Grainger

Veronica F. Grasso

Dariusz Grech

David R. Groholski

Oksana Grynyshyna-Poliuga

Marcin Grzesiak

Chen $\mathrm{Gu}$

Massimo Guerrero

Jinyong Gui

Laura Gulia

Tamara L. Gulyaeve

Thomas Günther

Leicheng Guo

Yuhang Guo

Ravi P. Gupta

Shivam Gupta

S. C. Gupta

Alexandra Guy
Ian Guymer

Man Ha Quang

John Bosco Habarulema

S. Hadiloo

Manouchehr Haghighi

Hojjat Haghshenas Lari

Bezalel C. Haimson

Hamid Haitham

Afera Halefom

Hossein Hamidifar

Hugo Hammer

Peng Han

Songjun Han

Paul Hardaker

Bruce S. Hart

K. Hasan

Youssef M. A. Hashash

Hossein Hashemi

Denis Hatzfeld

M. Hayakawa

Peng He

Gabi Hegerl

Oliver Heidbach

Kosuke Heki

Marcelo Henrique Leao-Santos

Frank Herrmann

Khaled Hessami

Richard Hey

C. Hibert

Dannie Hidayat

Gudrun Hillebrand

Gregor Hillers

Ali M. Hinis

Ekkehard Holzbecher

Catherine Homberg

Afshin Honarbakhsh

Sunhak Hong

Yang Hong

Young Hong Shin

Zhang Hongming

Sun Hongyu

Seyyed Behrouz Hosseyni

Matthew D. Howland

Sigrún Hreinsdóttir

Vagelis Hristidis

Nai-Chi Hsiao

Ta-Chih Hsiao

Bo Huang

Jianping Huang

Qinghua Huang

Shengzhi Huang

Weilin Huang

Zhen Huang
Christian Huggel

Allen Husker

Yunjung Hyun

Ismael M. Ibraheem

Adam Idziak

Iunio Iervolino

Olusegun Omoniyi Ige

Takeshi Iinuma

Nathan Intrator

Mansour Ioualalen

Mohsen Ahmadzadeh Irandoust

Trevor Irons

Tsuneo Ishido

Amir Ismail

Turgay İşseven

Ursula Iturrarán-Viveros

Marian Ivan

Yazad Jabbar

James Jackson

Forough Jafary

Alessandro Jaia

Frantisek Janda

Wojciech Jarmolowski

Jadwiga Jarzyna

Ravidra Jaybhaye

Maciej Jefimow

Solomon Jekayinfa

Vergne Jérôme

Madan K. Jha

WenHua Ji

Xiaofeng Jia

Zeyu Jia

Han Jiajun

Zhihong Jiang

Xinqi Jiao

A. Y. Jillo

Hong Jin

Lai Jin

Side Jin

Jorge Jódar

Ihab Jomaa

Ian Jones

Denis Jongmans

Geeta S. Joshi

Laurence Jouniaux

Waldemar Jozwiak

Niklas Juhojuntti

Tadeusz Juliszewski

Marek Kaczorowski

Diethelm Kaiser

Koosha Kalhor

I. I. Kalinnikov

John Kalogiros 
Thomas Kalscheuer

Osamu Kamigaichi

Masashi Kamogawa

Ali Kanli

Utku Kânoğlu

Vassilis Karakostas

Shawgar Karami

Emilia Karamuz

Abbaspour Karim

Subhankar Karmakar

Altin Karriqi

Dimitris Kaskaoutis

Elina Kasvi

Dimitris Katsanos

Richard W. Katz

Rennie B. Kaunda

Kirsti Kauristi

Y. Kawada

J. R. Kayal

Hossein Kazemi

Nasser Kazemi

Saskia Keesstra

Elliott Kellner

Ahmed Kenawy

Thomas Kenny

Perveiz Khalid

Qudrat Khan

Manoj Khandelwal

E. Alam Kherani

Malik Sikander Hayat Khiyal

Stefan Kienzle

Andrzej Kijko

Nazan Kilic

Hung Soo Kim

Sungwon Kim

Peter Kinnell

Marta Kiraga

A. Kiratzi

Kirill Kireyev

Ozgur Kisi

Timea Kiss

Bastian Klein

Zbigniew Klos

Krzysztof Kochanek

Silvia Kohnova

Kory Konsoer

Łukasz Kortas

Jari Kortström

Konrad Kossacki

Alex Kostinski

Vladimir Kostoglodov

Andrzej Kotyrba

Svetlana Kovacikova
Sebastian Kowalczyk

Richard Krahenbuhl

Vitold Krajeski

Adam Krajewski

T. Kronrod

Lech Krysinski

Wenhuan Kuang

Andrzej Kułak

Nagesh Kumar

Prashant Kumar

Rohini Kumar

Zbigniew Kundzewicz

Osamu Kuwano

Serdar Kuyuk

H. Serdar Kuyuk

Jeff Kwang

Annie O. L. Kwok

Eun Hye Kwon

John W. Labadie

Borys Ladanivskyy

A. V. Ladynin

Carlo Giovanni Lai

Jinxing Lai

Rattan Lal

Giovanni Lanzano

Stefano Lanzoni

Christophe Larroque

Anna Laskownicka

Francisco Latosinski

Alexander Lavrov

Jesse Lawrence

D. Lawton

Lazar Lazic

H. Le

Cheng-Haw Lee

Jae Min Lee

Saro Lee

Maximilien Lehujeur

Matthieu Lepot

Andrzej Lesniak

Paweł Leśniak

Hanna Levaniemi

Bailing Li

Elita Yunyue Li

Fangyu Li

Guilong Li

Haipeng Li

Jiaxuan Li

Jianzhu Li

Junlun Li

Rui Li

Ruopu Li

Zhanqing Li
Changhong Lin

Helen Lin

Hongbo Lin

Yu-Pin Lin

Larry Lines

V. A. Liperovsky

Chaofeng Liu

Jann-Yenq Liu

Jing Liu

Lanbo Liu

Mian Liu

Naihao Liu

Qiancheng Liu

Qingrui Liu

Shaoyong Liu

Wei Liu

Xiaobo Liu

Xiaozhou Liu

Yunfeng Liu

Anastasia Lobanova

Anna Łoboda

Tobias Lochbühler

Rowena B. Lohman

Monika Łój

Barbara Lolli

Fernando Lopez-Caballero

Nastaran Lotfi

Petroula Louka

Samuel Louvet

Jun Lu

Yang Lu

Eva Ludi

Steve Luis

Jewell Lund

Bin Luo

Jingrui Luo

Juhua Luo

Song Luo

Yiqi Luo

Lucia Luzi

Chao Ma

Jianquan Ma

Ming Ma

Yueling Ma

Dave MacLeod

M. Macouin

Nafiz Maden

Rafael Magallanes-Quintanar

Luis Fernando Magaña Solís

Viviana Maggioni

Rezene Mahatsente

Sharafeldin Mahmoud

Sahameddin Mahmoudi Kurdistani 
Mojtaba Mahsuli

Karen Mair

Mariusz Majdański

Marija Maksin

Luca Malagnini

Jacques Malavieille

Arash Malekian

Michal Malinowski

A. A. Malovichko

Vivek Manekar

Alessandro Mangione

Shattri Mansor

B. P. Marchant

David Marchant

Clezio Marcos De Nardin

Kurt J. Marfurt

Krzysztof Markowicz

Bertha Marquez Azua

Ivan Dimitri Marroquin

J. Marteau

J. D. Martínez-Vargas

Fatima Martin-Hernandez

Marta Martinkova

Cristina Martín-Moreno

Christian Massari

Demetrios Matsakis

Elena Matta

M. Mattei

Satya Maurya

Stefan Maus

Jessie Mayor

Maria Maza

Andrzej Mazur

Timothy McClanahan

Dan McEvoy

Helen McFarlane

Maurice J. Mchugh

Daniel E. McNamara

Brian J. O. L Mcpherson

Gerald Meehl

Nisar Memon

Banu Mena Cabrera

Arlindo Meque

Karina Merdith

Katrin Meusburger

Patrick Meyers

Milosz Mezyk

Siyu Miao

Mateusz Mikołajczak

Rasoul Mirabbasi

Vimal Mishra

Siddharth Misra

Krzysztof Mizerski
Mirka Mobilia

Victor Mocanu

Jamshid Moghadasi

Asoodeh Mojtaba

Roberto S. Molina Garza

Eugenio Molina Navarro

Juan Carlos Montalvo

Lorena Montoya

Seulgi Moon

Demie Moore

Max Moorkamp

Shahin Moradi

Isabelle Moretti

J. V. Morgan

Igor Morozov

Bill Morris

Jerzy Moscicki

Domiziano Mostacci

Mehdi Mousavi

Seyed Yaser Moussavi Alashloo

Isaac Mugume

Ehsan Muhammad Irfan

Babel Mukand

Birgit Müller

Asmita Murukumar

Michael Musgrove

Giovanni Musolino

Syed Md. Touhidul Mustafa

L. P. Muthuwatta

Grzegorz Mutke

Syed Fahad Naeem

Norihiro Nakamura

Jarek Napiorkowski

Balaji Narasimhan

Nadine Nassif

Jolanta Nastula

F. Alejandro Nava Pichardo

Ana D. Navarro-Ortega

Adeel Nazeer

Marina Nenković-Riznić

Mark New

Van Giang Nguyen

José Manuel Nicoalu Ibarra

Iacopo Nicolosi

Bogdan Niculescu

Jovana Nikolov

Rewati Niraula

Michi Nishioka

Edwin Nissen

Yaxiao Niu

Rosane Nobre

Pawel Nogas

Michael Nones
Vahid Nourani

Agata Novara

Izuchukwu Obiadi

Mathias Obrebski

Jide Nosakare Ogunbo

Philip Oguntunde

Matthias Ohrnberger

Seda Okay

Taikan Oki

Ilesanmi Oladapo

Michailovich Oleg V.

Gerrit Olivier

Nils Olsen

Per-Ivar Olsson

Oluwole Omosuyi

T. Ondoh

Mahir Öner

Sultan Ceren Öner

Victor N. Oparin

Emmanuel Opolot

Modesto Ortiz Figueroa

Mehdi Ostadhassan

Marzena Osuch

Gengxin $\mathrm{Ou}$

Dimitar Ouzounov

Elijah Oyeyemi

A. Arda Ozacar

Özden Özdemir

Amalio F. Pacheco

Francesca Pacor

ellora padhi

John Paine

Debasish Pal

Subodh Chandra Pal

Michele Palermo

Athanasios D. Panagopoulos

Panos Panagos

D. Panagoulia

Biranchi Panda

R. Pandey

Chris Paola

Maria Nicolina Papa

Georgia Papacharalampous

Eleftheria Papadimitriou

Juraj Parajka

Duhee Park

William Parton

J. N. Patel

Guy Paxman

Jana Pazdirkova

Song Peiping

José A. Peláez

Louise Pellerin 
Frederic Pellet

Zhigang Peng

Antonio Pepe

Deborah Pereg

Loredana Perrone

Claire Perry

Nils Perttu

Laura Peruzza

Moczo Peter

Andrew Pethick

Carole Petit

Gregor Petkovšek

Andrea Petroselli

Eduard Petrovsky

Huong Phan Thien

Melodie Philippon

Camilo Phillips

M. Picozzi

Adam Pidlisecky

R. Pielke

Jorge O. Pierini

Jan Pietron

Elżbieta Pilecka

Zenon Pilecki

Mark Pilkington

Santosh Pingale

Mikolaj Piniewski

Adam Piotrowski

M. A. PiRemli

Vladilen Pisarenko

Marta Pischiutta

Jacek Piskozub

Thomas Planes

T. Plenefisch

Boris Podobnik

Ina Pohle

Orhan Polat

Olga Polechonska

Piero Poli

Silvia Pondrelli

Felice Carlo Ponzo

Zbigniew Popek

Barbara Popielawska

Ryan Porter

Sławomir Porzucek

Hamid Reza Pourghasemi

Lubov Poustovalova

Biswajeet Pradhan

Yellapu Siva Prasad

Janire Prudencio

Anna Przebindowska

Stafano Pucci

David Pulido-Velázquez
Sergei Pulinets

Jehangir Punthakey

Hui Qian

Changyuan Qin

Tianling Qin

Xiaojun Qiu

Beatriz Quesada-Montano

Yoann Quesnel

Luis Quintanar

Shahid Nadeem Qureshi

Francesco Radaelli

Selvakumar Radhakrishnan

Prashanth Ragam

M. S. Rahman

Omid Rahmati

Rajkumar Raikar

Carlo Rainieri

Agnieszka Rajwa-Kuligiewicz

Hamidreza Ramazi

Leonardo Ramirez-Guzman

Shilpesh Rana

Trygve Randen

Eugene Rankey

Ying Rao

Sternfels Raphael

Grzegorz Raś

Dhananjay Ravat

Jan Reda

Julie Regnier

Julie Régnier

Nora Reichert

Carl Reine

Eduardo Reinoso Angulo

Rajesh Rekapalli

Liliang Ren

Peizhen Ren

Abbas Rezaei

Mohammad Rezaie-Balf

David Rhoades

Alessandra Ribodetti

Michael Riediker

Jaromír Říha

Joakim Riml

John Ristau

Vladica Ristić

Coen J. Ritsema

Monica Riva

Fabian Rivera

Enzo Rizzo

Gareth Roberts

Scott Robeson

Cordula Robinson

Steven Roche
Adrian Rodriguez-Marek

Jerzy Rojek

Vincenzo Romano

Renata Romanowicz

Sandoval-Florez Romulo

Wolfgang Rösler

Olga Rosowiecka

Zachary E. Ross

Matthias Roth

Hanna Rothkaehl

Renata Rotondi

Pawel Rowinski

Anna Rozwadowska

Youyi Ruan

Lucas Dominguez Ruben

Nils Ruther

Christopher Ruybal

Federico Sabina

Mauricio Sacchi

T. Sagiya

Pratap Narayan Sahay

Netrananda Sahu

Gaci Said

Anup Saikia

Pallabi Saikia

Aduwati Sali

Ali Sanati

Luiz Carlos Sanches

Francisco Sánchez-Sesma

Wenjing Sang

Hamid Sanginabadi

Langgeng Wahyu Santosa

Miguel Santoyo

Giulio Saracino

Alexandr Saraev

Coskun Sari

Sankar Sarkar

Nicholas Sarlis

Majid Sartaj

Claudio Satriano

Robert Sausen

Silvia Scarpetta

Ralf Schaa

Michael Scheuerer

Erik Schiefer

Stanisław Schillak

Simon Schmidt

Ingo Schnauder

Roman Schreiber

Kai Schröter

Maike Schumacher

Jeffrey Schweitzer

Laura Scognamiglio 
Emmanuel Scordilis

Christopher Scott

Carlo Scotto

Uma Seeboonruang

Gopi Seemala

Mrinal K Sen

Zekai Şen

I. P. Senanayake

Ye-Won Seo

M. Sepehr

Naya Servitzoglou

Anastasios G. Sextos

Umut Sezen

Amin Shaban

Mojtaba Shadmani

Munawar Shah

Tushaar Shah

Kalil Shahbazi

Shamsuddin Shahid

Guang-Zhou Shao

Lei Shao

Yaping Shao

Alan Shapiro

Arabinda Sharma

Babita Sharma

Alireza Shateri

Ranjit Shaw

Bin She

Yi Shen

Aleksey Y. Sheshukov

Peidong Shi

Ying Shi

Zheming Shi

Hiroshi Shinohara

Haan Shixing

David R. Shklyar

Saeed Shojaei

D. L. Shrestha

Da Shuai

James Shucksmith

Marcin Siepak

Fernando J. S. Silva Dias

Hengki Simanjuntak

Slobodan Simonovic

Chandrani Singh

Grzegorz Sinicyn

Michael Sioutas

Andreas Skarlatoudis

Agnieszka Skowron

Louise Slater

Michael Slawinski

Evert Slob

Łukasz Słonka
Maxim Smirnov

Fernando Soares Schlindwein

I. N. Sokolova

Jerzy Sobotka

Elena Yurievna Sokolova

Efthimios Sokos

Urszula Somorowska

Luis Somoza

Maxime Souvignet

Mike Spiliotis

Shalivahan Srivastava

Olivier Stab

Aaron Stanton

Ilias Stavrakas

Colby Steelman

Mchał Stefaniuk

Petra Štěpančíková

Jonathan P. Stewart

Simon Stewart

Stavroula Stolaki

Stelios Stoulos

F. O. Strasser

Cristine Stumpp

Zhongbo Su

K. P. Sudheer

Ettammal Suhas

Jiajia Sun

Qiang Sun

Wenyi Sun

Nicola Surian

Adi Suryadi

Rupert Sutherland

Norikazu Suzuki

Susan K. Swanson

Arsalan Syed

Norbert Szabo

Dawid Szafranski

Artur Szkop

Jan Szolgay

Malgorzata Szwed

Adam Szymkiewicz

T. J. P. M. Op t Root

Hossein Tabari

Ali Rashid Tabrez

Isomura Takuya

Ali Talebi

Morteza Talebian

Elcin Tan

Mou Leong Tan

Hiroyuki K. M. Tanaka

Jizhou Tang

Gulum Tanircan

Dan Tao
Toyoizumi Taro

Matteo Taroni

Mohammad Tatar

R. E. Tatevossian

Claudia Tebaldi

Jacek Tejchman

Luciano Telesca

Donatella Termini

Asirat Teshome

Pankaj Thapa

Nikolaos Theodoulidis

Wrona Thilo

Brian Thomas

Brian F. Thomas

Renie Thomas

Nan Tian

Larisa Tichtchenko

Dieu Tien Bui

Timo Tiira

Fred Tillman

Elisa Tinti

Cenk Toker

S. K. Tomar

Michał Tomczak

François Tondeur

Tomasz Topor

Joan Miguel Torta

Ivana Tosic

Vincenzo Totaro

Roberta Tozzi

Markos D. Tranos

Jorg Trentmann

Simon Tresch

Jui-Pin Tsai

Alexandros Tsavdaris

Ioannis M. Tsodoulos

Kagan Tuncay

Endre Turai

Azra N. Tutuncu

Serhat Tüzün

Tomasz Tyminski

Evangelos Tyrlis

Andreas Tzanis

Trushit Upadhyay

Andrzej Urbaniec

A. Uromeihy

Ruben Urraca

Zbigniew Ustrnul

Babak Vaheddoost

Rein Vaikmae

Katri Vaittinen

Carlos Valdés González

Jan Valenta 
Raúl Valenzuela Wong

Le Van Anh Cuong

Henny A. J. Van Lanen

Anne Van Loon

R. D. Van Remortel

Cees Van Westen

Andres Vargas-Luna

Emmanouil Varouchakis

Arianna Varrani

Lampros Vasiliades

Ivana Vasiljevic

Massimiliano Vassallo

Saeed Vatankhah

Arie Vatresia

Sreeja Veettil

Ramesh Vellore

Kalyana C. Veluvolu

Sumit Verma

D. J. Verschuur

Foteini Vervelidou

Davide Vettori

W. W. S. Vieira

Daniele Viero

Olga Vigiak

Giulio Vignoli

Francesco Visini

Elena Volpi

James Voogt

Sergiy Vorogushyn

M. T. Vu

Richard Walker

Clemens Walther

Yunxia Wan

Niko Wanders

Di Wang

Hao Wang

Hua Wang

Hui Wang

Kun Wang

Meng Wang

Shifeng Wang

Tieyi Wang

Xiangchun Wang

Yuefeng Wang

Arnaud Watlet

Kamila Wawrzyniak-Guz

Shimon Wdowinski

Fabian Welc

Kuo-Liang Wen

Yixin Wen

Friedemann Wenzel
Lukasz Wiejaczka

Simon D. P. Williams

Peter Wintoft

Piotr Witek

Axel Wittmann

Edwin Wnuk

Kurt Wogau

Marek Wojdyla

Nam C. Woo

Andrew W. Wood

Anders Worman

Daniel Wright

Bangyu Wu

Jianchao Wu

Rushan Wu

Wei Wu

Wensheng Wu

Yao Wu

Yih-Min Wu

Zhonghai Wu

Zhongliang $\mathrm{Wu}$

Jobst Wurl

Gilead Wurman

C. Wylie Poag

Max Wyss

Andrzej Wyszogrodzki

Bartłomiej Wyżga

Stelios Xanthos

Liang Xiao

Maohua Xiao

Tao Xu

Youpeng Xu

Yungui Xu

Shibei Xue

R. N. Yadav

Sanjay Yadav

Soheil Yahyapour

Farzam Yaminifard

Denghua Yan

Jidong Yang

Qingjie Yang

Qinke Yang

Gang Yao

Morgan Yarker

Zaher Yaseen

Nergis Yasmin

Eric Yee

Yi-Lung Yeh

Emrah Yenier

C. Yonezawa
Heesung Yoon

Jiachun You

Jinsheng You

Qingyu You

Isma Younes

E. G. Youngs

Guo Yu

Peng Yu

Yongcai Yu

Sanyi Yuan

Zainuddin M. Yuso

Hasan Zabihi

Hamidreza Zahabi

I. E. Zakharenkova

Ahmad Zamani

Eddy Y. Zeng

Hongyu Zhai

Bo Zhang

Chao Zhang

Chong Zhang

Hao Zhang

Rui Zhang

Ruili Zhang

Shaohua Zhang

Wei Zhang

Xiaoping Zhang

Xiong Zhang

Yi-chun Zhang

Yijie Zhang

Zhen Zhang

Guangju Zhao

Peiqiang Zhao

Yan Zhao

Yang Zhao

Xiaowei Zhao

Yingcai Zheng

Xiefei Zhi

Jian Zhou

Shiyong Zhou

M. Zhu

Peimin Zhu

Rixiang Zhu

Senlin Zhu

Marcin Zielinski

George Zittis

Bogdan Zogala

Matthew Zook

Tomasz Zorski

Joseph Zume

Ramon Zuñiga

We apologize for any errors or inadvertent omissions. 